International Journal of Theoretical and Applied Finance Vol. 10, No. 8 (2007) 1365-1368

(C) World Scientific Publishing Company

\title{
Author Index Volume 10 (2007)
}

Abutaleb, A. \& Papaioannou, M. G., Malliavin Calculus for the Estimation of TimeVarying Regression Models Used in Financial Applications

Al-Zoubi, H. A. \& Maghyereh, A. I., The Relative Risk Performance of Islamic Finance: A New Guide to Less Risky Investments

An, Y., Assaf, A. \& Yang, J., Hedging Volatility Risk: The Effectiveness of Volatility Options

Arai, T., An Approximate Approach to the Exponential Utility Indifference Valuation

Assaf, A., see An, Y.

Baxter, M., Lévy Simple Structural Models

Bordag, L. A. \& Chmakova, A. Y., Explicit Solutions for a Nonlinear Model of Financial Derivatives

Boyarchenko, N. \& Levendorskiǐ, S., On Errors and Bias of Fourier Transform Methods in Quadratic Term Structure Models

Branger, N. \& Schlag, C., Option Betas: Risk Measures for Options

Brigo, D., Pallavicini, A. \& Torresetti, R., ClusterBased Extension of the Generalized Poisson Loss Dynamics and Consistency with Single Names

Brody, D. C., Constantinou, I. C. \& Meister, B. K., Term Structure of Vanilla Options

Buchmann, B. \& Weber, S., A Continuous Time Approximation of an Evolutionary Stock Market Model

5 (2007) 771

Campolieti, G. \& Makarov, R., Pricing PathDependent Options on State Dependent Volatility Models with a Bessel Bridge

2 (2007) 235

3 (2007) 517

3 (2007) 475

3 (2007) 517

4 (2007) 593

$1(2007) 1$

2 (2007) 273

7 (2007) 1137

$4(2007) 607$

8 (2007) 1323
Ceccacci, S., Marchesiani, A. \& Pecchi, L., Public Debt Management and Foreign Currency Denominated Bonds

Chapovsky, A., Rennie, A. \& Tavares, P., Stochastic Intensity Modeling for Structured Credit Exotics

Chaudhary, S. K., A Simple American Option Pricing Method Using the Fast Fourier Transform

Chiarella, C., Nikitopoulos Sklibosios, C. \& Schlögl, E., A Markovian Defaultable Term Structure Model with State Dependent Volatilities

Chmakova, A. Y., see Bordag, L. A.

Christensen, M. M. \& Platen, E., Sharpe Ratio Maximization and Expected Utility When Asset Prices Have Jumps

Chu, C. C. \& Kwok, Y. K., Valuation of Guaranteed Annuity Options in Affine Term Structure Models

Ciprian, M., see d'Addona, S.

Clémençon, S. \& Slim, S., On Portfolio Selection Under Extreme Risk Measure: The Heavy-Tailed ICA Model
7 (2007) 1229

$1(2007) 51$

5 (2007) 763

4 (2007) 633

7 (2007) 1191

1 (2007) 155

1 (2007) 1

8 (2007) 1339

2 (2007) 363

2 (2007) 307

3 (2007) 449 
Constantinou, I. C., see Brody, D. C.

Costa, A., see Krzesinski, A. E.

d'Addona, S. \& Ciprian, M., Time Varying Sensitivities on a Grid Architecture

d'Addona, S., see Marinelli, C.

Dash, J. W., Multivariate Integral Perturbation Techniques. I: Theory

Davis, M. H. A. \& Esparragoza-Rodriguez, J. C., Large Portfolio Credit Risk Modeling

Dempster, M. A. H., Medova, E. A. \& Yang, S. W., Empirical Copulas for CDO Tranche Pricing Using Relative Entropy

Dondi, G., see Herzog, F.

Elouerkhaoui, Y., Pricing and Hedging in a Dynamic Credit Model

Epple, F., Morgan, S. \& Schloegl, L., Joint Distributions of Portfolio Losses and Exotic Portfolio Products

Esparragoza-Rodriguez, J. C., see Davis, M. H. A

Fabozzi, F. J. \& Tunaru, R., On Some Inconsistencies in Modeling Credit Portfolio Products

Fahrner, I., Modern Logarithms for the Heston Model

Francis, J. C., see Harel, A.

Geering, H. P., see Herzog, F.

Geering, H. P., see Keel, S.

Gürtler, M. \& Hartmann, N., The Equity Premium Puzzle and Emotional Asset Pricing

Györfi, L., Urbán, A. \& Vajda, I., Kernel-Based Semi-Log-Optimal Empirical Portfolio Selection Strategies

Harel, A. \& Harpaz, G., Fair Actuarial Values for Deductible Insurance Policies in the Presence of Parameter Uncertainty

Harel, A., Harpaz, G. \& Francis, J. C., Pricing Securities with Exchange-Imposed Price Limits via Risk Neutral Valuation
8 (2007) 1323

1 (2007) 89

2 (2007) 307

6 (2007) 1043

8 (2007) 1287

4 (2007) 653

4 (2007) 679

2 (2007) 203

4 (2007) 703

4 (2007) 733

4 (2007) 653

8 (2007) 1305

1 (2007) 23

3 (2007) 399

2 (2007) 203

6 (2007) 1015

6 (2007) 939

3 (2007) 505

2 (2007) 389

3 (2007) 399
Harpaz, G., see Harel, A.

Harpaz, G., see Harel, A.

Hartmann, N., see Gürtler, M.

He, Z.-W., see Zhu, S.-P.

Herzog, F., Dondi, G. \& Geering, H. P., Stochastic Model Predictive Control and Portfolio Optimization

Herzog, F., see Keel, S.

Hikspoors, S. \& Jaimungal, S., Energy Spot Price Models and Spread Options Pricing

Hubalek, F. \& Sgarra, C., Quadratic Hedging for the Bates Model

Huehne, F., Defaultable Lévy Libor Rates and Credit Derivatives

Ikonen, S. \& Toivanen, J., Componentwise Splitting Methods for Pricing American Options Under Stochastic Volatility

Ishii, D., see Yamamoto, R.

Jacob, M. J., see Thampi, K. K.

Jaimungal, S., see Hikspoors, $\mathrm{S}$.

Keel, S., Herzog, F., Geering, H. P. \& Schumann, L. M., Optimal Portfolio Construction Under Partial Information for a Balanced Fund

Konno, H., see Yamamoto, R.

Korn, R. \& Kovilyanskaya, H., A General Framework for High Yield Bond Investment

Kovilyanskaya, H., see Korn, R.

Krzesinski, A. E., Costa, A., Ramakrishnan, M. \& Taylor, P. G., A Comment on Two-Phase Behavior of Financial Markets

Kwok, Y. K., see Chu, C. C.

Levendorskiǐ, S., see Boyarchenko, N.

Maghyereh, A. I., see AlZoubi, H. A.

Makarov, R., see Campolieti, G.

Marchesiani, A., see Ceccacci, S.

Marinelli, C., d'Addona, S. \& Rachev, S. T., A
2 (2007) 389

3 (2007) 399

6 (2007) 939

7 (2007) 1203

2 (2007) 203

6 (2007) 1015

7 (2007) 1111

5 (2007) 873

3 (2007) 407

2 (2007) 331

6 (2007) 1095

5 (2007) 837

7 (2007) 1111

6 (2007) 1015

6 (2007) 1095

6 (2007) 967

6 (2007) 967

1 (2007) 89

2 (2007) 363

2 (2007) 273

$2(2007) 235$

1 (2007) 51

5 (2007) 763 
Comparison of Some Univariate Models for Valueat-Risk and Expected Shortfall

Medova, E. A., see Dempster, M. A. H.

Meister, B. K., see Brody, D. C.

Mishra, A. K., The Market Reaction to Stock Splits - Evidence from India

Mollah, A. S., Testing WeakForm Market Efficiency in Emerging Market: Evidence from Botswana Stock Exchange

Morgan, S., see Epple, F.

Nayak, S., An EquilibriumBased Model of StockPinning

Ng, S.-A., Margin Trading Through Hyper Timeline

Nikitopoulos Sklibosios, C., see Chiarella, C.

Njoh, S., Cross Hedging within a Log Mean Reverting Model

Ostrovsky, D., BlackScholes-Merton in Random Time: A New Stochastic Volatility Model with Path Dependence

Ostrovsky, D., Constant Elasticity of Variance in Random Time: A New Stochastic Volatility Model with Path Dependence and Leverage Effect

Pallavicini, A., see Brigo, D.

Pan, X., The Linear Dependence and Feedback Spectra Between Stock Market and Economy

Papaioannou, M. G., see Abutaleb, A.

Pecchi, L., see Ceccacci, S.

Platen, E., see Christensen, M. M.

Rachev, S. T., see Marinelli, C.

Raju, N., see Thampi, K. K.

Ramakrishnan, M., see Krzesinski, A. E.

Rennie, A., see Chapovsky, A.

Rutkowski, M. \& Yousiph, K., PDE Approach to the Valuation and Hedging of Basket Credit Derivatives

Rutkowski, M. \& Yu, N., An Extension of the Brody-Hughston-

6 (2007) 1043

4 (2007) 679

8 (2007) 1323

2 (2007) 251

6 (2007) 1077

4 (2007) 733

3 (2007) 535

5 (2007) 801

1 (2007) 155

5 (2007) 887

$5(2007) 847$

6 (2007) 915

4 (2007) 607

3 (2007) 437

5 (2007) 771

5 (2007) 763

8 (2007) 1339

6 (2007) 1043

5 (2007) 837

1 (2007) 89

$4(2007) 633$

8 (2007) 1261
Macrina Approach to Modeling of Defaultable Bonds

Schlag, C., see Branger, N.

Schloegl, L., see Epple, F.

Schlögl, E., see Chiarella, C.

Schumann, L. M., see Keel, $\mathrm{S}$.

Sgarra, C., see Hubalek, F.

Shiraishi, H. \& Taniguchi, M., Statistical Estimation of Optimal Portfolios for Locally Stationary Returns of Assets

Sidenius, J., On the Term Structure of Loss Distributions: A Forward Model Approach

Slim, S., see Clémençon, S.

Soumaré, I., Equilibrium with Excessive Holdings Constraint: An Application to DC Pension Plans

Souza, M. O. \& Zubelli, J. P., On the Asymptotics of Fast Mean-Reversion Stochastic Volatility Models

Stace, A. W., A Moment Matching Approach to the Valuation of a Volume Weighted Average Price Option

Taniguchi, M., see Shiraishi, $\mathrm{H}$.

Tavares, P., see Chapovsky, A.

3 (2007) 557

7 (2007) 1137

4 (2007) 733

1 (2007) 155

6 (2007) 1015

5 (2007) 873

1 (2007) 129

4 (2007) 749

3 (2007) 449

7 (2007) 1159

$5(2007) 817$

1 (2007) 95

$1(2007) 129$

$4(2007) 633$

$1(2007) 89$ A. E.

Thampi, K. K., Jacob, M. J. \& Raju, N., Barrier Probabilities and Maximum Severity of Ruin for a Renewal Risk Model

Toivanen, J., see Ikonen, S.

Torresetti, R., see Brigo, D.

Tunaru, R., see Fabozzi, F. J.

Urbán, A., see Györfi, L.

Vajda, I., see Györfi, L.

Vardas, G. \& Xepapadeas, A., Uncertainty Aversion, Robust Control and Asset Holdings with a Stochastic Investment Opportunity Set

6 (2007) 985

Weber, S., see Buchmann, B.

Xepapadeas, A., see Vardas,

5 (2007) 837

2 (2007) 331

4 (2007) 607

8 (2007) 1305

3 (2007) 505

3 (2007) 505

7 (2007) 1229

6 (2007) 985 
Yamamoto, R., Ishii, D. \& Konno, H., A Maximal Predictability Portfolio Model: Algorithm and Performance Evaluation

Yang, H., A New Finite Element Method for Pricing of Bond Options Under Time Inhomogeneous Affine Term Structure Models of Interest Rates

Yang, J., see An, Y.

Yang, S. W., see Dempster, M. A. H.
Yousiph, K., see Rutkowski, M.

8 (2007) 1261

Yu, N., see Rutkowski, M.

Zhang, J. E., see Zhu, Y.

3 (2007) 557

1 (2007) 111

6 (2007) 1095

Zhu, S.-P. \& He, Z.-W., Calculating the Early Exercise Boundary of American Put Options with an Approximation Formula

Zhu, Y. \& Zhang, J. E., Variance Term Structure and VIX Futures Pricing

3 (2007) 517

4 (2007) 679 Zubelli, J. P., see Souza, M. O. 